\title{
Protocol for a cluster randomised controlled trial of LPG cookstoves compared to usual cooking practices to reduce perinatal mortality and morbidity in rural Bangladesh called Poriborton: The CHANge Trial.
}

Camille H Raynes-Greenow ( $\nabla$ camille.raynes-greenow@sydney.edu.au ) University of Sydney https://orcid.org/0000-0002-8802-6226

\section{Ashraful Alam}

The University of Sydney

Sk Masum Billah

ICDDR: International Centre for Diarrhoeal Disease Research Bangladesh

Sajia Islam

ICDDR B: International Centre for Diarrhoeal Disease Research Bangladesh

Kingsley Agho

Western Sydney University

\section{SM Rokonuzzaman}

ICDDR: International Centre for Diarrhoeal Disease Research Bangladesh

Jonathan Thornburg

RTI International

\section{Shams El Arifeen}

ICDDR: International Centre for Diarrhoeal Disease Research Bangladesh

\section{Atique Iqbal Chowdhury}

ICDDR: International Centre for Diarrhoeal Disease Research Bangladesh

\section{Michael J Dibley}

The University of Sydney

\section{Research Article}

Keywords: Indoor air pollution, perinatal mortality, Bangladesh, cluster randomised controlled trial.

Posted Date: February 1st, 2022

DOI: https://doi.org/10.21203/rs.3.rs-948333/v1 
License: (c) (i) This work is licensed under a Creative Commons Attribution 4.0 International License. Read Full License

Version of Record: A version of this preprint was published at Trials on April 18th, 2022. See the published version at https://doi.org/10.1186/s13063-022-06146-7. 


\section{Abstract}

\section{Background}

Household air pollution is a leading health risk for global morbidity and mortality and a major health risk in South Asia. However, there are no prospective investigations of the impact of household air pollution on perinatal morbidity and mortality. Our trial aims to assess the impact of liquefied petroleum gas (LPG) for cooking to reduce household air pollution exposure on perinatal morbidity and mortality compared to usual cooking practices in Bangladesh.

Hypothesis:

In a community-based cluster randomised controlled trial of pregnant women cooking with LPG throughout pregnancy, perinatal mortality will be reduced by $35 \%$ compared with usual cooking practices in a rural community in Bangladesh.

\section{Methods}

A two-arm community-based cluster randomised controlled trial in the Sherpur district, Bangladesh. In the intervention arm, pregnant women receive an LPG cookstove and LPG in cylinders supplied throughout pregnancy until birth. In the control or usual practice arm, pregnant women continue their usual cooking practices, predominately traditional stoves with biomass fuel. Eligible women are pregnant women with a gestational age of 40 - 120 days, aged between 15 and 49 years, and permanent residents of the study area. The primary outcome is the difference in perinatal mortality between the LPG arm and the usual cooking arm. Secondary outcomes include i) preterm birth and low birth weight, ii) personal level exposure to household air pollution, iii) satisfaction and acceptability of the LPG stove and stove use, iv) cost-effectiveness and cost-utility in reducing perinatal morbidity and mortality. We follow up all women and infants to 45 days after the birth. Personal exposure to household air pollution is assessed at threetime points in a sub-sample of the study population using the MicroPEM $^{\text {TM }}$. The total required sample size is 4,944 pregnant women.

\section{Discussion}

This trial will produce evidence of the effectiveness of reduced exposure to household air pollution through LPG cooking to reduce perinatal morbidity and mortality compared to usual cooking practices. This evidence will inform policies for the adoption of clean fuel in Bangladesh and other similar settings.

Trial registration:

Prospectively registered with the Australian New Zealand Clinical Trials Registry, ACTRN12618001214224, 19th July 2019, www.anzctr.org.au.

\section{Version $12^{\text {th }}$ Sept 2021}




\section{Background}

Globally, household air pollution is a leading health risk for mortality, contributing up to approximately 4.3 million deaths or $7.7 \%$ of all deaths per year $[1,2]$. Approximately 3 billion people depend on solid fuels (e.g. wood, dung, crop waste) for cooking and this combined with incomplete combustion using inefficient stoves, in poorly ventilated areas, over long hours of cooking and heating, causes exposure to household air pollution [3]. The South Asian burden is acute, with approximately $74 \%$ of households depending on solid fuels for cooking [4]. The established disease profile includes acute respiratory infection, ischaemic heart disease and lung cancer [2]. However, there are no comprehensive investigations or confirmation of the impact of household air pollution on pregnancy outcomes [5]. Pregnant women and children are at greater risk due to a combination of their higher vulnerability and their higher levels of exposure due to domestic responsibilities [6-8]. There is some epidemiological evidence of the effect of household air pollution on perinatal outcomes, including three published systematic reviews $[5,8,9]$. The most recent review examined the impact on stillbirth and low birth weight [9]. The reported summary-effect estimate on stillbirth was 1.29 (95\% Cl 1.18, 1.41), and for low birth weight, $1.35(95 \% \mathrm{Cl} 1.23,1.48)[9]$. Overall, all reviews report low quality of the included studies due to the mostly retrospective designs with a high risk of information and selection bias, unclear definitions of stillbirth with small sample sizes, considerable variability in the exposure assessment, insufficient adjustment for confounders, and the most recent review noted evidence of publication bias [8-12]. The association for early neonatal mortality is also not clearly understood from these studies.

Perinatal mortality is a major global public health problem $[13,14]$. Globally, neonatal mortality and stillbirth are extraordinarily high, with approximately 2.5 million global neonatal deaths a year and $\sim 2.6$ million stillbirths per year [13]. South Asia has the second-highest stillbirth burden and accounts for almost $40 \%$ of all stillbirths[13]. In Bangladesh, the stillbirth rate is 35 per 1000 births, and worse in rural areas $[15,16]$, where there is almost universal reliance on polluting fuels [17-19].

In low resource settings, studies quantifying household air pollution exposure have reported pollutant levels in houses that far exceed the WHO acceptable levels [20]. In Bangladesh, exposure to biomass fuel emissions from cooking is very high, especially in rural settings. Three studies from Bangladesh, using data from the Bangladesh Demographic and Health Surveys, have described the use of solid fuel for cooking as high as $93 \%$, and associations between exposure from cooking with solid fuels and adverse perinatal outcomes such as stillbirth [21, 22], early neonatal mortality [22] and low birth weight [23]. Cleaner fuels such as liquefied petroleum gas (LPG) are generally and relatively available, scalable and considered an acceptable intervention to reduce exposure to household air pollution [24]. We developed a community-based cluster randomised controlled trial to assess the impact of cooking with LPG compared to usual cooking (predominantly traditional biomass fuel stoves) on perinatal mortality in pregnant women in rural Bangladesh. The trial is called Poriborton: The CHANge trial (Poriborton a Bangla word meaning 'change for better', and Clean Household Air for Newborns trial, ACTRN12618001214224). 


\section{Methods}

\section{Aims and hypothesis}

The primary aim is to assess the impact of LPG cooking in reducing perinatal mortality compared to usual cooking practices in rural Bangladesh. The secondary aims are to evaluate the effect of LPG cooking compared to usual cooking on neonatal mortality, preterm birth, and low birth weight. Our objective is to assess the reduction of exposure to household air pollution in the LPG cooking arm compared to usual cooking and determine the cost-effectiveness of LPG cooking compared to usual cooking in reducing perinatal morbidity and mortality.

We hypothesise that in a community-based, cluster randomised controlled trial of women in early pregnancy from a rural community in Bangladesh, the use of LPG will reduce perinatal mortality (stillbirth and early neonatal mortality) by $35 \%$ compared with usual cooking practices. We defined stillbirth from $\geq 28$ weeks gestation and early neonatal mortality up to seven days after birth[25].

\section{Study design}

The design is a community-based cluster randomised controlled trial (CRCT) of two arms. The intervention arm: i) includes an LPG cookstove and LPG cylinders with behaviour change communication, provided from early pregnancy until the birth and ii) the control arm, continues usual cooking practices. The usual cooking practice in this setting is a traditional clay stove with various biomass fuels [26].

\section{Study setting}

The study will be in the Sherpur district of Mymensingh Division, Bangladesh (Figure 1). Sherpur is approximately 200 kilometres north of Dhaka. The district has been selected based on the high neonatal mortality rates (46 per 1000 live births) and the low penetration of LPG fuel for cooking[19]. Sherpur has five sub-districts and a population of $\sim 1,170,219$ living in rural areas. Out of the five sub-districts, we will purposively select two for the study based on the road conditions that allow a good connection to the district headquarters for LPG transportation and study staff access. A total of 16 Unions (the smallest administrative unit) will be selected from the two chosen sub-districts considering: a) the community reliance on traditional cooking practices, b) geographically not prone to flooding during the monsoonal season, and c) a similar intervention is not ongoing in that area.

[insert figure 1 here]

\section{Design, randomisation and intervention allocation}

We define a cluster as a geographical area of $\sim 445$ households or $\sim 1800$ people. According to the 2011 population census of Bangladesh [27], the average population size per union in Sherpur is $\sim 25,000$ people yielding up to 20 clusters per Union. The field team will conduct an initial listing of residents in the study area to get the exact size of the population. We will then select 206 clusters and apply a block randomisation to allocate the clusters with a 1:1 ratio[28], resulting in 103 clusters per arm and an equal 
number of intervention and control clusters from each union. We will generate random blocks of sequential 1 and 2 using Stata SE[29], where each number represents either the intervention or control arm. Non-investigators will allocate the clusters to the treatment arms based on the random sequence.

The intervention is distributed at the individual level, but randomised at the cluster level to reduce community tensions and overall exposure, and leverage community-level adoption of clean cooking. We will conduct outcome assessment at the individual level. Allocation concealment is impractical due to the cluster design and nature of the intervention. Pregnant women and their households receive the intervention based on their cluster of residence, and LPG cylinder supply will continue through to birth (Figure 2).

[Insert figure 2 here]

\section{Study population}

We have two levels of inclusion and exclusion criteria, and the first level is for cluster eligibility. Eligible clusters are those with a predominantly rural population. Exclusion criteria are i) clusters already participating in a stove scheme or are within or sharing boundaries of areas with a relatively high existing use of LPG cooking, and ii) clusters prone to flooding for extended periods. The second level for eligibility is for women. Eligible women are i) between 15 to 49 years of age, ii) pregnant with a gestational age between 40 - 120 days determined by self-reported date of the last menstrual period, iii) permanent residents of the study area and iv) planning to give birth in their cluster of residence. Exclusion criteria include i) women whose gestational age cannot be determined, ii) pregnancy loss before receipt of the intervention and, iii) women who are not permanent residents of the study area.

\section{Feasibility study}

Before finalising the trial design, we conducted a single-arm non-randomised feasibility study of LPG stove and cylinder distribution [30]. The findings from this study had multiple outcomes that identified issues and improved processes for conducting our trial. Including the development a locally appropriate LPG cylinder supply chain in the study area. Development of content relevant for our initial behaviour change communication such as benefits of clean cooking, practical suggestions for modifying cooking behaviours, and the important role of mothers-in-law and husbands in decision making about cooking and fuel practices[30]. Lastly, we confirmed culturally appropriate approaches for measuring personal exposure to household air pollution, and produced representative data that confirmed the LPG intervention did reduce exposure to cooking fuel emissions (manuscript in preparation).

\section{Formative research}

We conducted formative research to more specifically inform the behaviour change communication materials. Experienced qualitative researchers trained in ethnographic methods stayed in selected households in the trial area to observe the cooking behaviour of the family. The researcher collected extensive field notes of their observations and conducted interviews with the person mainly responsible 
for cooking in the family to generate data on families' everyday cooking behaviours and related aspects, current cooking practices, and the role of persons within the family and the community in cooking-related decision-making. In addition, we performed direct observation of the cooking environment, including the kitchen and any other places used for cooking and fuel storage. We conducted focus groups with women to generate information about the women's perceptions and experiences of usual cooking practices and adopting LPG stoves for cooking. This was done to develop culturally appropriate and attractive messages for the community to support consistent and correct use of LPG cooking during the intervention period (manuscript in preparation).

\section{Intervention}

The LPG stove will have a double burner, and we will use a $15 \mathrm{~kg}$ cylinder, and the Bangladesh Standard and Testing Institute have accredited both stoves and cylinders. The stove company provides logistic support, maintenance, and technical troubleshooting throughout the study period. Our field implementation team are trained in technical issues related to gas stove set-up, ignition, connection and use, troubleshooting, maintenance, and cylinder replacement.

The behaviour change communication messages are designed to promote and support individual and household adoption of correct and consistent LPG cooking. The intervention field staff will distribute the behaviour change communication material and support the participants and their household members in the intervention arm once a month to promote consistent use of LPG cooking during pregnancy. They will also discuss practical stove use issues and safety. The materials include a calendar and a leaflet with pictorial messages and three short movies, plus a fourth focused solely on safety played on a tablet device. The scripts for the short movies will be iteratively written in a local dialect by our team and professionally produced by the gas supplier using actors local to the study site. These movies will follow the story of a household that would be considered typical in our study area who purchase an LPG stove and cylinder for the newly pregnant woman and will be performed by local actors in a local dialect. Each movie is a slightly different story for each pregnancy trimester and will run for approximately 5-10 minutes.

To ensure continuous LPG coverage, we have collaborated with the LPG supplier to establish a supply chain for the project. The participants will request an LPG replacement cylinder via our intervention worker (either by phone or in-person) who enters the request into the web-based system specifically developed for this trial, which will be accessible in the field using electronic tablets. The intervention worker will then forward the notification of the request to the respective gas storage hub manager (there are approximately 1-2 of these per Union). The participant or her representative will collect the replacement cylinder from the hub manager within 24 hours after the initial request, which we will track using our real-time tablet system.

\section{Identification and enrolment of eligible women and supply of LPG stove and cylinder}


We will have three types of field workers for study implementation: married women surveillance staff, intervention staff and evaluation (outcome) staff. The surveillance staff will identify eligible women and conduct door-to-door visits to the households in both the intervention and control areas in two monthly cycles. In the initial round, the surveillance staff will prepare a comprehensive list of all existing currently married women in the study clusters (pregnancy outside marriage is not common in the study area). They will then prospectively visit the listed married women every alternate month until the required number of pregnancies are identified and enrolled from each cluster. At each contact, currently married women of reproductive age will report their last menstrual cycle date. Any woman whose date of her last menstrual cycle is $>40$ days will be recorded as potentially pregnant. Pregnancy will be confirmed by a urine test strip kit (Exel®). Once a woman is identified and recorded as pregnant on our web-based data collection platform, the system will send an automatic notification to the evaluation worker. The evaluation worker will visit the pregnant woman and, following written informed consent, will enrol the woman. A list of all enrolled women from the intervention clusters will be sent to the intervention facilitators. Within 72 hours of the enrolment notification, the intervention facilitators will visit the women to provide detailed information on the gas stove, introduce the participant or her representative to the local gas storage hub manager and explain the process of collecting the stove and cylinder. The intervention facilitators will also ask participants to prepare a place for installing the stove. On the next visit, made within seven days of enrollment, the intervention facilitators will demonstrate the LPG stove and cylinder installation and inform enrolled women of the process for subsequent gas cylinder replenishment. In this visit, the intervention facilitators will counsel the women and other household members regarding safety measures and share the behaviour chance communication material. The visits will continue monthly.

\section{Improve adherence and monitor compliance}

The monthly visit by the intervention facilitators is to support exclusive use of LPG cooking, ensure safety and troubleshoot any issues. The tasks consist of observing the cooking place with a checklist (including looking for signs of biomass cooking), delivery of the behaviour change material, and monitor safety. If evidence of biomass fuel cooking is detected our facilitators will discuss the reason and encourage the woman and her household to cook exclusively with LPG.

\section{Management and training}

A team of four field supervisors will monitor the $\sim 70$ surveillance staff and ten intervention facilitators and report to a field Research Manager to supervise all the field activities. We will train all staff in their respective duties for the household visit and pregnancy identification. Intervention facilitators will receive intensive training in installing and using LPG, and behaviour change messages related to exclusive use of gas for cooking. We will conduct the training in phases, with regular refresher courses, and monthly meetings to discuss of field-related troubleshooting. Supervisors will also provide daily feedback to the staff.

\section{Control arm}


Study participants do not receive a cookstove or LPG cylinders in the control arm and continue their usual cooking practices. Usual cooking practices in this setting and most rural settings in Bangladesh involve a traditional clay stove with an open fire burning biomass fuel, usually crop waste and sticks. However, this varies by season and household income. Evidence of the effectiveness of improved stoves in the field suggested that they were unlikely to sufficient reduce household air pollution and we therefore did not consider them an appropriate comparator[31]. Control participants will receive a small gift at the birth visit (blanket).

\section{Outcomes and assessment of the outcomes}

\section{Primary outcomes}

The primary outcome is perinatal mortality rate differences between the LPG and the usual cooking arm. Perinatal mortality is a composite indicator, including the sum of the number of stillbirths (fetal death in pregnancy of at least 28 weeks gestation or more) and the number of early neonatal deaths (the death of a live-born infant up to and including six days of age regardless of gestational age or birth weight) [25, 32].

\section{Secondary outcomes}

Our secondary outcomes include a comparison between intervention and control arm in terms of i) perinatal morbidity: preterm birth (birth before 37 completed weeks gestation or in the absence of gestation birth weight <2500grams) and low birth weight (<2500gm); ii) cost-effectiveness of LPG in reducing perinatal mortality; iii) personal level exposure to household air pollution; iv) satisfaction and acceptability of the LPG stove and stove use (per cent of cooking time with LPG stove), and vi) costeffectiveness and cost-utility of gas cooking compared to the usual cooking practice. We will also measure changes between groups at each time point in blood pressure, anaemia and gestational diabetes. We anticipate that women in the LPG group will be less hypertensive, less anemic; however, we do not expect differences in gestational diabetes.

We capture the pregnancy outcome data in both study arms within 48 hours of birth, perinatal mortality up to 15 days after the birth, and neonatal deaths up to 45 days after the birth.

\section{Exposure assessment of particulate matter}

Personal household air pollution exposure will be measured in a subset of 300 women equally divided between each arm. Data collection will occur at 3-4 months gestation, 5-6 months and 8-9 months. We will use the MicroPEM (RTI, Research Triangle Park, NC) worn in the pocket of a sash located on the upper left of the women's chest to measure personal exposure. The MicroPEM is a low burden and wearable (240 g with batteries) particulate matter (PM) exposure monitor. $\mathrm{PM}_{2.5}$ concentrations will be measured with a nephelometer and with a filter. Additional filter analysis will quantify the black carbon and tobacco smoke concentration [33]. We calculated the estimated participant compliance with wearing the MicroPEM from the accelerometer data[34]. Purple Air monitors deployed outdoors will measure ambient 
$\mathrm{PM}_{2.5}$ concentrations to separate HAP exposures from other exposure sources such as brick kilns or rice mills.

\section{Process evaluation for adequacy of the intervention}

Data on cooking practices are collected at baseline using a structured questionnaire and visual inspection (with a checklist) of the cooking environment, which we have used before[26, 35]. Adherence to LPG cooking is collected monthly in the intervention arm. This data will identify any contextual factors that influence (exclusive) use of LPG cooking [36]. Qualitative data is collected through interviews and focus groups of a purposively selected sample of intervention recipients (women and their household members) and all cadres of project staff.

\section{Sample size and power calculation}

We calculated the sample size for the primary outcome, a reduction in the perinatal mortality rate in the intervention compared to the usual practice arm. A systematic review of polluting fuels versus clean fuels reported an elevated risk of perinatal mortality of 56\% (aRR:1.44, 95\% Cl: 1.30, 1.61) [37]. Therefore, we have calculated our sample size conservatively informed by this estimate, and the considered data from published studies at the time of developing the protocol $[5,8,11,12]$. We assumed a $35 \%$ decrease in perinatal mortality (effect size) in the LPG arm. Our previous field work estimated perinatal mortality at 50 per 1000 pregnancies in control clusters[38]. We considered 80\% power and 5\% two-sided alpha and an average cluster size of 20 births. The published intra-class correlations for stillbirth and neonatal mortality in rural Bangladesh are very low, 0.00055 and 0.00000 respectively [39]; thus, we used a conservative intra-class correlation midway between the estimates of 0.000275[39]. We used the standard formula [40] to estimate the number of births required for the study to be 4,120 (2,060 per arm) from a total of 206 clusters. We inflated the sample size to attain these births to 4944 pregnant women (2,472 per arm) from a total of 206 clusters ( 24 women per cluster) considering 10\% pregnancy loss (abortion/miscarriage < 28 weeks gestation) and another loss of 5\% of women who move and birth outside the study area.

\section{Sample size for the household air pollution exposure}

We used the data from our feasibility study and a previous study conducted in Sri Lanka to estimate the sample size for the personal exposure measurements [41]. The total number needed is 300 women (150 per arm) to complete the personal exposure measurements at three gestational time points. This will demonstrate a reduction in household air pollution exposure resulting from the LPG intervention with an effect size of 0.5 and $95 \%$ power and $5 \%$ error.

\section{Data collected}

We will collect demographic, socioeconomic, household characteristics, birth history, birth outcomes, birthweight, maternal anthropometry, cost of care-seeking, cooking practices, and sources of household air pollution surveys from all participating women. We will also assess the haemoglobin and non-fasting 
glucose. These are collected with standard field practices using a drop of blood from a needle prick and measured with an hemocue or glucometer. Measurements will be taken at baseline and in the final trimester 8-9 months gestation. We measure diastolic and systolic blood pressure using the CRADLE VSA at baseline, 4-5 months, at 8-9 months. Personal exposure to household air pollution will be collected as previously described. Figure 1 outlines the data collection schedule, and data collection forms available on request.

\section{Data collection procedures}

A separate team of $\sim 12$ evaluation staff with no involvement in trial implementation will be responsible for collecting the outcome assessment data. All evaluation staff are female and recruited locally and supervised by four field supervisors. All evaluation staff will receive training for two weeks with regular refresher training. Training will include all aspects of data collection, including consent taking, different data collection methods, anthropometric measurements, blood pressure measurement, haemoglobin and glucose, household air pollution exposure measurement, electronic data capture, and relevant cultural considerations. We will provide supportive supervision for all staff on site.

\section{Electronic data collection tool}

Data is captured electronically in the field using an android platform tablet with a specifically designed application (app). The app will record all surveillance and trial information. It will schedule visits for each household and prepare monthly visits and activity schedules for each data collector. The app reports all data back to the central database system in real-time, but the intervention and evaluation teams only have access to the data relevant to their position. The field research manager, overseen by staff in Dhaka monitors all field staff visit schedules and data quality.

\section{Access to data}

Data will be accessible by all investigators to analyse and publish., and we will only use the information from the respondents for research purposes. We will share data after removing participants' personally identifiable information. The participant personal identifiers entered by the data collector will automatically be suppressed from the data collection form once the data is uploaded to the passwordprotected server.

\section{Data management}

All electronic data is stored on a server backed-up at icddr,b and only limited staff can access it with passwords. Data will be stored for 15 years as per funder rules by the sponsor.

\section{Statistical analysis}

We will conduct an intention-to-treat analysis, and assess outcomes at the individual level. To evaluate the balance of key background and potential confounding variables, we will summarise the baseline characteristics by treatment group. To assess the impact of the intervention, we will compare perinatal 
mortality, preterm birth, and low birth weight between the intervention versus the control arm. Our analyses will estimate relative risks and $95 \%$ confidence intervals of point estimates using generalised estimating equations, logistic binomial regression models, a log link function, and exchangeable correlation [42]. The intervention will be a fixed effect, and the cluster as a random effect to account for clustering. We will do separate models for our specified outcomes. To ensure we only detect potentially important interactions with the mortality outcomes, we will increase power $(P<0.10(v s<0.05))$ in the generalised estimating equation logistic regression models [43]. There are no interim analyses planned and there are no stopping rules.

For the exposure data, we will calculate exposure-response functions for different exposure metrics, first using graphical summaries (e.g. loess plots) to examine the shape of the relationship of the exposure metrics to the outcome. For this initial exploratory stage, we will also use standard multivariate approaches (e.g. principal components, spectral decompositions) to reduce the dimensionality of highdimensional metrics. Real-time measurements of $\mathrm{PM}_{2.5}$ will allow us to explore which summary metrics (e.g., integrated total mass exposure, peak exposure levels, and time above risk threshold) are most closely associated with the outcome. Based on the exploratory analyses, we will then use appropriate generalised linear models to generate estimates of the effect of the exposure metrics (or combinations of) on the outcomes. We will calculate the effect sizes and $95 \%$ confidence intervals for all analyses and present the exact P-value.

\section{Trial registration}

We registered the study with the Australian New Zealand Clinical Trials Registry ACTRN12618001214224.

\section{Dissemination plan}

We will share the trial findings with the stakeholders from Bangladesh and (virtual) global audiences through a dissemination ceremony (if possible in person). We will also present the findings at conferences and publish in conference papers and international peer-reviewed journals. We will distribute the results using appropriate methods, such as community meetings. If the results are favourable to the intervention, we will work with relevant stakeholders to ensure access to the intervention for all communities, these discussions have already commenced.

This protocol has been written according to the recommendations of the SPIRIT 2013 statement[44], figure 4 .

\section{Adjustment to the procedures due to COVID-19 and status update}

In the last week of March 2020, the Government of Bangladesh instigated a movement restriction intervention to curb SARS-COV-2 infections, effective from the 26th of March, 2020. At that time, we had already finished our fifth round of household menstrual surveillance. We, therefore, stopped recruitment and decided not to enrol any new participants from the 26th of March. However, we continued household 
menstrual surveillance over the phone to allow the field team to quickly enrol eligible women once the restrictions were lifted. We also continued follow-up of the enrolled women over the phone. We made the necessary changes in the questionnaire; for instance, we added an additional question whether the visit was conducted physically or over the phone, and an extra option "Cannot be done as the interview was conducted over the phone" to the questions for maternal and newborn anthropometry. Our staff gave support for consistent and continued LPG use over the phone, and we sought clearance and were approved to continue LPG cylinder replacement during the lockdown.

We developed an Infection Prevention and Control protocol, based on WHO advice. We developed different infection protocols for each cadre of workers (i.e. office staff, surveillance workers, evaluation workers, intervention workers). Additionally, we are keeping a record of COVID-19 cases at the study site. Any staff who conduct home visits are also expected to adhere to a comprehensive Personal Protective Equipment protocol, and icddr,b has approved both protocols. All project staff received training on both protocols. From the 9th of August, 2020, we resumed enrollment of participants, physical visits for data collection and intervention facilitation. Field supervisors monitor the activities closely so that all protocols are strictly maintained. The field team reports to investigators on COVID-19 updates, the project staff's infection control procedures, and participants (if any) every week. All staff were fully vaccinated in early 2021.

At the time of submission of the manuscript all participants had been recruited and follow-up of the pregnant women was continuing and all births were expected to end in May 2022.

\section{Ethics approval and consent to participate}

The Research Review Committee and the Ethical Review Committee of icddr,b (PR-17103), and the Human Research Ethics Committee of the University of Sydney (PR-2018/717), Australia, approved the study. We have gate-keeper consent from community leaders and relevant government officials. All women and or their guardians provide informed consent to participate and have the right to withdraw at any stage without penalty or loss. The investigators will ensure the privacy, anonymity and confidentiality of the information provided by respondents, and will store all trial information in an encrypted database with all identifiers removed. We will report any Serious Adverse Event or unanticipated problems of potential risk for study participants or others to the relevant committees in writing within 24 hours of such occurrences. These will be monitored by the relevant committees and based on advice we will not establish a data monitoring committee. Any changes to the protocol will be communicated to the committees above, and the trial registry.

\section{Discussion}

This trial will provide high-level of evidence of the effect of reduced exposure to household air pollution from cooking with LPG on pregnancy outcomes, specifically perinatal mortality and morbidity. Until now, evidence of the effect on perinatal mortality has been mostly observational, retrospective and or using secondary data, and for perinatal studies, neither the exposure nor perinatal mortality have been 
measured accurately and, or prospectively, leaving uncertainty around the estimates [9]. Accurately measuring perinatal morality even in high-burden countries requires a prospective design, with an accurate case definition that considers the context of the setting and is sufficiently powered to detect a difference. Small, retrospective studies are generally insufficiently powered, and more importantly, are subject to recall bias.

The Government of Bangladesh released a strategic plan for LPG distribution, and it declared a target of $70 \%$ for all households to use LPG by 2030 . They previously set access to electricity targets for 2021 , but these did not cover cooking fuels[45-47]. Clean or cleaner fuel targets are important for health and the environment and Goal seven of the Sustainable Development Goals [48] includes such targets. This trial will provide evidence of the impact of household air pollution on perinatal outcomes.

\section{Trial status}

Protocol version one (19 $9^{\text {th }}$ July 2019). Recruitment commenced in Sept 2019, and ended in June 2021, follow-up is expected to be complete in May 2022. There have been several hurdles in running the trial due to the pandemic, including confirmed SARS-CoV-2 infections in our implementing team and movement restriction orders placed on staff in three countries (Bangladesh, Australia and the US). These and the changes to our roles and responsibilities due to these movement restrictions (home-schooling children, online teaching, increased workload, inability to travel to the study site, etc) have adversely impacted our available time, and progress, and hence we submit this protocol after the recruitment was completed.

\section{Declarations}

\section{Consent for publication}

All authors have approved the final version and provide their consent for publication.

\section{Availability of data and materials}

Not relevant

\section{Competing interests}

All authors report no competing interests.

\section{Funding and roles}

The National Health and Medical Research Council (Australia) GNT1027074 funds the project. The initial protocol development occurred when CRG held an Academy of Science (Australia), Australia-India ECR Fellowship. The protocol completion occurred during a Career Development Fellowship supported by the NHMRC_GNT1087062. The trial sponsor is The University of Sydney, contact details are available from 
the corresponding author. Neither the sponsor or the funders have any role or decisions in any activities. The LPG gas supplier has no authority in the study beyond cylinder supply, and movie production as described above. They have requested their name not to be reported. All data will be accessible to the study investigators, and will have the right to analyse and publish data.

\section{Authors' contributions}

CRG developed the original protocol with support from MJD, with relevant expertise from all authors and the Poriborton trial team. AA developed the formative protocol and the process evaluation; JT developed the exposure assessment protocol. SMB principally led the field implementation plan. Field activities were overseen by SI, with SMR, and with advice from SEA and other experts at icddr,b included in the Poriborton Trial Team.

\section{Acknowledgements}

We wish to acknowledge the women and children in Sherpur who have helped shape this protocol. Elisa Puzzolo also provided advice on LPG supply and introduced us to relevant contacts, for which we are grateful. The Poriborton Trial Team includes the authors listed, and the full team includes those and additionally Bin Jalaludin, Nick Goodwin, Alison Hayes, Tanvir Huda, Md. Jahiduj Jaman, and Jasmin Khan.

\section{References}

1. Lim SS, Vos T, Flaxman AD, Danaei G, Shibuya K, Adair-Rohani H, AlMazroa MA, Amann M, Anderson $\mathrm{HR}$, Andrews KG: A comparative risk assessment of burden of disease and injury attributable to 67 risk factors and risk factor clusters in 21 regions, 1990-2010: a systematic analysis for the Global Burden of Disease Study 2010. The lancet 2013, 380(9859):2224-2260.

2. Gakidou E, Afshin A, Abajobir AA, Abate KH, Abbafati C, Abbas KM, Abd-Allah F, Abdulle AM, Abera SF, Aboyans $V$ et al: Global, regional, and national comparative risk assessment of $\mathbf{8 4}$ behavioural, environmental and occupational, and metabolic risks or clusters of risks, 1990-2016: a systematic analysis for the Global Burden of Disease Study 2016. The Lancet 2017, 390(10100):1345-1422.

3. WHO: Air Quality Guidelines Global Update 2005: Particulate matter, ozone, nitrogen dioxide and sulfer dioxide. In.: WHO; 2005.

4. Krishna B, Balakrishnan K, Siddiqui AR, Begum BA, Bachani D, Brauer M: Tackling the health burden of air pollution in South Asia. Bmj 2017, 359:j5209.

5. Pope DP, Mishra V, Thompson L, Siddiqui AR, Rehfuess EA, Weber M, Bruce NG: Risk of Low Birth Weight and Stillbirth Associated With Indoor Air Pollution From Solid Fuel Use in Developing Countries. Epidemiologic Reviews 2010, 32(1):70-81.

6. Bruce N, Dherani M, Das JK. etal.: Control of household air pollution for child survival: estimates for intervention impacts. BMC Public Health 2013, 13(Suppl 3). 
7. WHO: Indoor air pollution from solid fuels and risk of low birth weight and stillbirth: report from a symposium held at the Annual Conference of the International Society for Environmental Epidemiology (ISEE), September 2005, Johannesburg. In.: World Health Organization; 2007.

8. Tielsch JM, Katz J, Thulasiraj RD, Coles CL, Sheeladevi S, Yanik EL, Rahmathullah L: Exposure to indoor biomass fuel and tobacco smoke and risk of adverse reproductive outcomes, mortality, respiratory morbidity and growth among newborn infants in south India. Int J Epidemio/2009, 38(5):1351-1363.

9. Amegah AK, Quansah R, Jaakkola JJK: Household Air Pollution from Solid Fuel Use and Risk of Adverse Pregnancy Outcomes: A Systematic Review and Meta-Analysis of the Empirical Evidence. PLOS ONE 2014, 9(12):e113920.

10. Mavalankar DV, Trivedi CR, Gray RH: Levels and risk factors for perinatal mortality in Ahmedabad, India. Bulletin of the World Health Organization 1991, 69(4):435-442.

11. Mishra V, Retherford RD, Smith KR: Cooking smoke and tobacco smoke as risk factors for stillbirth. International Journal of Environmental Health Research 2005, 15(6):397-410.

12. Siddiqui A, Gold E, Brown K, Lee K, Bhutta Z: Preliminary analyses of indoor air pollution and low birth weight (LBW) in Southern Pakistan. In: Indoor air pollution from solid fuels and risk of low birth weight and stillbirth Report from a symposium held at the Annual Conference of the International Society for Environmental Epidemiology (ISEE): 2005; Johannesburg, South Africa: 2005; 2005: 11 14.

13. Lawn JE, Blencowe H, Waiswa P, Amouzou A, Mathers C, Hogan D, Flenady V, Frøen JF, Qureshi ZU, Calderwood C: Stillbirths: rates, risk factors, and acceleration towards 2030. The Lancet 2016, 387(10018):587-603.

14. Hug L, Alexander M, You D, Alkema L: National, regional, and global levels and trends in neonatal mortality between 1990 and 2017, with scenario-based projections to 2030: a systematic analysis. The Lancet Global Health 2019, 7(6):e710-e720.

15. Cousens S, Blencowe H, Stanton C, Chou D, Ahmed S, Steinhardt L, Creanga AA, Tunçalp Ö, Balsara ZP, Gupta S: National, regional, and worldwide estimates of stillbirth rates in $\mathbf{2 0 0 9}$ with trends since 1995: a systematic analysis. The Lancet 2011, 377(9774):1319-1330.

16. Ellis M, Azad K, Banerjee B, Shaha SK, Prost A, Rego AR, Barua S, Costello A, Barnett S: Intrapartumrelated stillbirths and neonatal deaths in rural Bangladesh: a prospective, community-based cohort study. Pediatrics 2011, 127(5):e1182-e1190.

17. Bangladesh Government: Bangladesh Country Action Plan (CAP) for Scaling Clean Cooking Solutions. In. Dhaka: Ministry of Power Division; 2013.

18. Raynes-Greenow CH, Dibley MJ, El-Arifeen S, Alam A, Agho K, T H, S I, on behalf of the Shonjibon trial study team: The impact of household air pollution on perinatal mortality; results from a large embedded cohort study in a rural setting in Bangladesh. In: Society of Pediatric and Perinatal Epidemiology Annual Meeting (June 2016). Miami, Florida; 2016. 
19. National Institute of Population Research and Training (NIPORT) MaA, and ICF International: Bangladesh Demographic and Health Survey 2014. In. Dhaka, Bangladesh, and Rockville, Maryland, USA: NIPORT, Mitra and Associates, and ICF International; 2016.

20. Gurley ES, Salje H, Homaira N, Ram PK, Haque R, Petri Jr WA, Bresee J, Moss WJ, Luby SP, Breysse P et al: Seasonal concentrations and determinants of indoor particulate matter in a low-income community in Dhaka, Bangladesh. Environmental Research 2013, 121(0):11-16.

21. Khan MN, B Nurs CZ, Mofizul Islam M, Islam MR, Rahman MM: Household air pollution from cooking and risk of adverse health and birth outcomes in Bangladesh: a nationwide population-based study. Environmental health : a global access science source 2017, 16(1):57-57.

22. Nisha MK, Alam A, Raynes-Greenow C: Variations in perinatal mortality associated with different polluting fuel types and kitchen location in Bangladesh. International Journal of Occupational and Environmental Health 2018, 24(1-2):47-54.

23. Haider MR, Rahman MM, Islam F, Khan MM: Association of Low Birthweight and Indoor Air Pollution: Biomass Fuel Use in Bangladesh. Journal of Health and Pollution 2016, 6(11):18-25.

24. Amegah AK, Jaakkola JJK: Household air pollution and the sustainable development goals. Bulletin of the World Health Organization 2016, 94:215-221.

25. Blencowe H, Calvert PC, Lawn JE, Cousens S, Campbell OMR: Measuring maternal, foetal and neonatal mortality: Challenges and solutions. Best Practice \& Research Clinical Obstetrics \& Gynaecology 2016, 36:14-29.

26. Sk Masum Billah, Sajia Islam, Fariha Tasnim, Ashraful Neeloy Alam, Shams El Arifeen, RaynesGreenow CH: Self-adopted 'natural users' of liquid petroleum gas for household cooking by women in pregnancy in rural Bangladesh: characteristics of high use and opportunities for intervention. Environ Res Lett 2020, in press https://doi.org/10.1088/1748-9326/ab7b25.

27. BANGLADESH BUREAU OF STATISTICS (BBS), STATISTICS AND INFORMATICS DIVISION (SID), MINISTRY OF PLANNING GOTPSRO, BANGLADESH: Population and housing census 2011. In.; 2012.

28. Efird J: Blocked randomization with randomly selected block sizes. International journal of environmental research and public health 2011, 8(1):15-20.

29. StataCorp. 2017. Stata Statistical Software: Release 15. College Station, TX: StataCorp LLC. In.

30. Raynes-Greenow C, Islam S, Khan J, Tasnim F, Nisha MK, Thornburg J, Billah SM, Alam, Ashraful: A Feasibility Study Assessing Acceptability and Supply Issues of Distributing LPG Cookstoves and Gas Cylinders to Pregnant Women Living in Rural Bangladesh for Poriborton: The CHANge Trial. International Journal of Environmental Research and Public Health 2020, 17(3):848.

31. Kyle Steenland, Ajay Pillarisetti, Miles Kirby, Jennifer Peel, Maggie Clark, Will Checkley, Howard H Chang, Clasen T: Modeling the potential health benefits of lower household air pollution after a hypothetical liquified petroleum gas (LPG) cookstove intervention. Environment internationa/ 2017, 111:71-79.

32. World Health Organization: International Statistical Classification of Diseases and Related Health Problems - 10th revision. In., vol. 2, 2010 edn. Geneva: World Health Organization; 2011. 
33. Lawless P, Rodes C, Ensor D: Multiwavelength absorbance of filter deposits for determination of environmental tobacco smoke and black carbon. Atmospheric Environment 2004, 38:3373-3383.

34. Lawless P, Thornburg J, Rodes $C$, Williams R: Personal exposure monitoring wearing protocol compliance: An initial assessment of quantitative measurement. Journal of Exposure Science \& Environmental Epidemiology 2012, 22(3):274-280.

35. Alam A, Tawale N, Patel A, MJ D, Jadhao S, CH R-G: Household Air Pollution intervention implications: findings from qualitative studies and a field trial of clean cookstoves in two rural villages in India. (Accepted Aug 2016 International Journal of Environmental Research and Public Health) 2016

36. Lawless PA, Rodes CE, Ensor DS: Multiwavelength absorbance of filter deposits for determination of environmental tobacco smoke and black carbon. Atmospheric Environment 2004, 38(21):3373-3383.

37. Patel AB, Meleth S, Pasha O, Goudar SS, Esamai F, Garces AL, Chomba E, McClure EM, Wright LL, Koso-Thomas M: Impact of exposure to cooking fuels on stillbirths, perinatal, very early and late neonatal mortality-a multicenter prospective cohort study in rural communities in India, Pakistan, Kenya, Zambia and Guatemala. Maternal health, neonatology and perinatology 2015, 1(1):18.

38. Huda TM, Rahman MM, Raihana S, Islam S, Tahsina T, Alam A, Agho K, Rasheed S, Hayes A, Karim MA et al: A community-based cluster randomised controlled trial in rural Bangladesh to evaluate the impact of the use of iron-folic acid supplements early in pregnancy on the risk of neonatal mortality: the Shonjibon trial. BMC Public Health 2018, 18(1):816.

39. Pagel C, Prost A, Lewycka S, Das S, Colbourn T, Mahapatra R, Azad K, Costello A, Osrin D: Intracluster correlation coefficients and coefficients of variation for perinatal outcomes from five clusterrandomised controlled trials in low and middle-income countries: results and methodological implications. Trials 2011, 12(1):151.

40. Murray DM: Design and analysis of group-randomized trials, vol. 29: Oxford University Press, USA; 1998.

41. Chartier R, Phillips M, Mosquin P, Elledge M, Bronstein K, Nandasena S, Thornburg V, Thornburg J, Rodes C: A comparative study of human exposures to household air pollution from commonly used cookstoves in Sri Lanka. Indoor Air 2016:n/a-n/a.

42. Liang K-Y, Zeger SL: Longitudinal data analysis using generalized linear models. Biometrika 1986:1322.

43. West KP, Shamim AA, Mehra S, Labrique AB, Ali H, Shaikh S, Klemm RD, Wu LS, Mitra M, Haque R: Effect of maternal multiple micronutrient vs iron-folic acid supplementation on infant mortality and adverse birth outcomes in rural Bangladesh: the JiVitA-3 randomized trial. Jama 2014, 312(24):26492658.

44. Chan A-W, Tetzlaff JM, Gøtzsche PC, Altman DG, Mann H, Berlin JA, Dickersin K, Hróbjartsson A, Schulz KF, Parulekar WR et al: SPIRIT 2013 Explanation and Elaboration: Guidance for protocols of clinical trials. BMJ 2013, 346:200-207. 
45. 70\% households to get LPG in 3 years [http://www.dhakatribune.com/bangladesh/2016/10/27/70households-get-lpg-3-years/]

46. Taheruzzaman M, Janik P: Electric Energy Access in Bangladesh. Transactions on Environment and Electrical Engineering 2016, 1.

47. https://data.worldbank.org/indicator/EG.ELC.ACCS.ZS?locations=BD

48. https://www.un.org/sustainabledevelopment/energy/

\section{Figures}

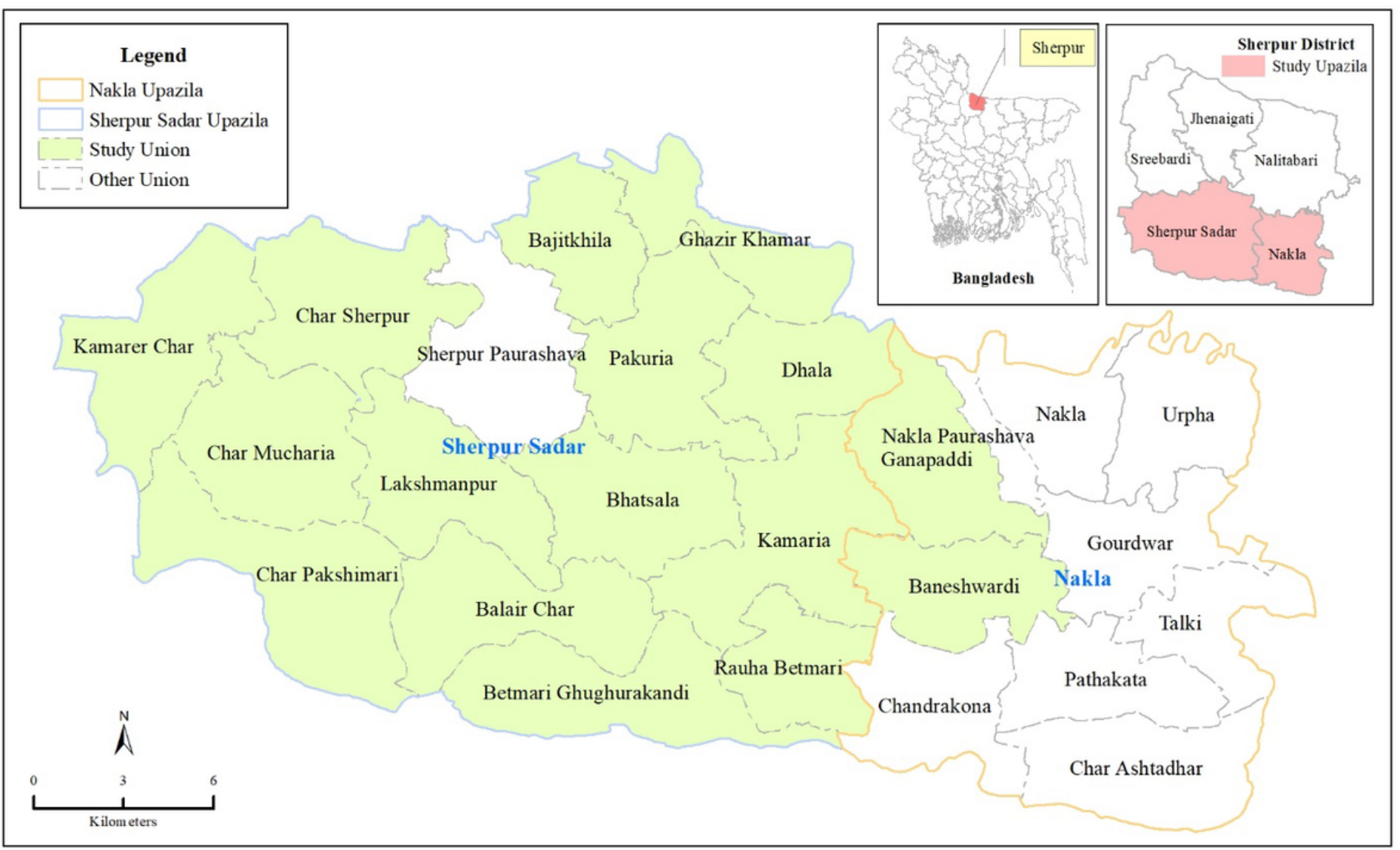

Figure 1

Sherpur, the Poriborton study site, in Northern Bangladesh, divided by Unions (main picture). Insert: Bangladesh divided by Districts, and the selected Sub-Districts (red). 


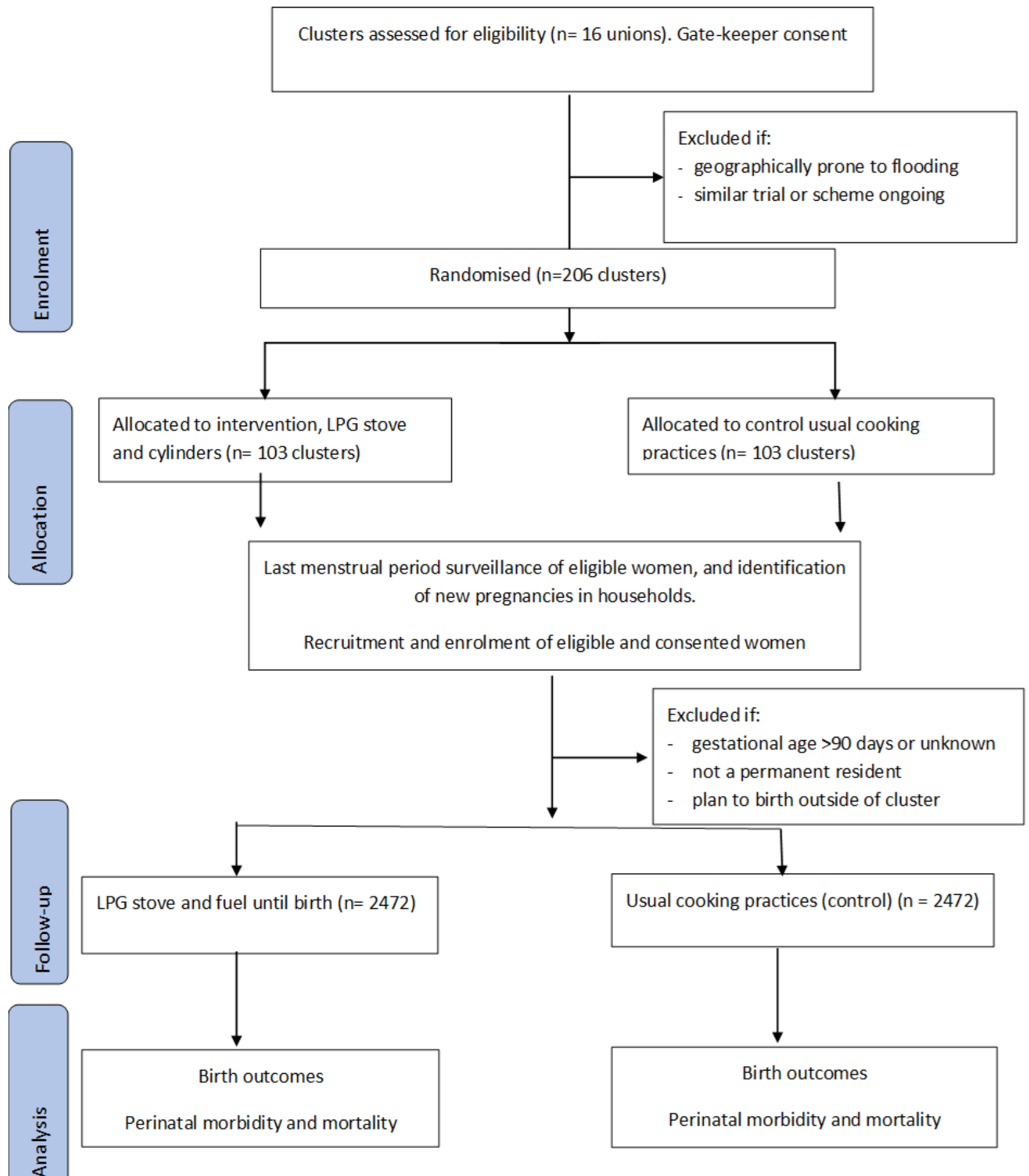

\section{Figure 2}

Poriborton trial flow diagram. 


\begin{tabular}{|c|c|c|c|c|c|c|}
\hline \multirow{3}{*}{ Time Point } & \multicolumn{6}{|c|}{ STUDY PERIOD } \\
\hline & \multicolumn{3}{|c|}{ Gestational age (months) } & \multicolumn{2}{|l|}{ Birth } & \multirow{2}{*}{\begin{tabular}{|l|} 
Neonata \\
Period \\
$\mathbf{4 5}^{\text {th }}$ day \\
\end{tabular}} \\
\hline & 3-4 & $5-6$ & $8-9$ & 48 hours & $7-10$ days & \\
\hline \multicolumn{7}{|l|}{ ENROLMENT: } \\
\hline Eligibility and enrolment & $\mathrm{x}$ & & & & & \\
\hline Informed consent & $\mathrm{X}$ & $\mathrm{X}$ & & & & \\
\hline \multicolumn{7}{|l|}{ INTERVENTION } \\
\hline \multicolumn{7}{|l|}{ Arm $1 L P G$} \\
\hline \multicolumn{7}{|l|}{ Arm 2 Control } \\
\hline \multicolumn{7}{|l|}{ ASSESSMIENT } \\
\hline \multicolumn{7}{|l|}{ Background characteristics } \\
\hline $\begin{array}{l}\text { All background characteristics } \\
\text { (socioeconomic status, } \\
\text { education, etc.) }\end{array}$ & $\mathrm{X}$ & & & & & \\
\hline Pregnancy history & $\mathrm{X}$ & & & & & \\
\hline \multicolumn{7}{|l|}{ Pregnancy and Birth Care } \\
\hline Maternal complication & $\mathrm{X}$ & $\mathrm{X}$ & $\mathrm{X}$ & & $\mathrm{X}$ & \\
\hline $\begin{array}{l}\text { Antenatal care related } \\
\text { information }\end{array}$ & $\mathrm{X}$ & $\mathrm{X}$ & $\mathrm{X}$ & & $\mathrm{X}$ & \\
\hline Cost of care-seeking & & $\mathrm{X}$ & $\mathrm{X}$ & & $\mathrm{X}$ & \\
\hline Birth outcome & & & & $\mathrm{X}$ & & \\
\hline Birth complication data & & & & & $\mathrm{X}$ & \\
\hline Birth care-seeking cost & & & & & $\mathrm{X}$ & \\
\hline PNC related data & & & & & $\mathrm{X}$ & \\
\hline Cost of (other) care seeking & & & & & $\mathrm{X}$ & \\
\hline $\begin{array}{l}\text { Essential Newborn Care } \\
\text { related data }\end{array}$ & & & & & $\mathrm{X}$ & \\
\hline $\begin{array}{l}\text { Exposure to household air } \\
\text { pollution }\end{array}$ & $\mathrm{X}$ & & & & & \\
\hline Cooking practices & $\mathrm{X}$ & $\mathrm{X}$ & $\mathrm{X}$ & & $\mathrm{X}$ & \\
\hline $\begin{array}{l}\text { Household air pollution } \\
\text { exposure data (by interview) }\end{array}$ & $\mathrm{X}$ & $\mathrm{X}$ & $\mathrm{X}$ & & $\mathrm{X}$ & \\
\hline $\begin{array}{l}\text { Household air pollution } \\
\text { exposure level measurement } \\
\text { (MicroPEM) }\end{array}$ & $\mathrm{X}$ & $\mathrm{X}$ & $\mathrm{X}$ & & $\mathrm{X}$ & \\
\hline $\begin{array}{l}\text { St George's Respiratory } \\
\text { Ouestionnaire }\end{array}$ & $\mathrm{X}$ & $\mathrm{X}$ & $\mathrm{X}$ & & $\mathrm{X}$ & \\
\hline \multicolumn{7}{|l|}{$\begin{array}{l}\text { Birth outcome and newborn } \\
\text { mortality }\end{array}$} \\
\hline Birth outcome & & & & $\mathrm{X}$ & & \\
\hline Pregnancy termination & & $\mathrm{X}$ & $\mathrm{X}$ & & & \\
\hline Preterm Birth & & & & $\mathrm{X}$ & & \\
\hline $\begin{array}{l}\text { Perinatal and newborn } \\
\text { mortality }\end{array}$ & & & & $\mathrm{X}$ & $\mathrm{X}$ & \\
\hline Neonatal complication & & & & $\mathrm{X}$ & $\mathrm{X}$ & \\
\hline$E Q-5$ d tool & $\mathrm{X}$ & $\mathrm{X}$ & $\mathrm{X}$ & $\mathrm{X}$ & $\mathrm{X}$ & $\mathrm{X}$ \\
\hline \multicolumn{7}{|l|}{ Anthropometry } \\
\hline Mother's height & $\mathrm{X}$ & & & & & \\
\hline Mother's weight & $\mathrm{X}$ & $\mathrm{X}$ & $\mathrm{X}$ & & & \\
\hline Child's birth weight & & & & $\mathrm{X}$ & & \\
\hline \multicolumn{7}{|l|}{ Maternal health } \\
\hline Haemoglobin measurement & $\mathrm{X}$ & & $\mathrm{X}$ & & & \\
\hline Blood Pressure & $\mathrm{X}$ & $\mathrm{X}$ & $\mathrm{X}$ & & $\mathrm{X}$ & \\
\hline Glucose measurement & $\mathrm{X}$ & & $\mathrm{X}$ & & & \\
\hline
\end{tabular}

\section{Figure 3}

Schedule of data collection and trial activities for Poriborton 


\section{Figure 4 is available in the Supplemental Files section}

\section{Figure 4}

\section{Supplementary Files}

This is a list of supplementary files associated with this preprint. Click to download.

- SPIRITchecklistporibortonSept2021.docx 